per litre, was allowed to drain and dried at $100^{\circ} \mathrm{C}$. The benzoates when chromatographed on this paper were revealed in ultra-violet light as dark spots on a yellow fluorescent background, a spot containing $10^{-8} \mathrm{gm}$. mole being just visible. Spots containing more than $10^{-6} \mathrm{gm}$. mole tend to overload the paper.

The normal technique of descending irrigation was slightly modified in order to maintain a saturated atmosphere in the chamber with the volatile solvents used. The chamber lid, fitted with a rubber gasket and a thumbscrew tightening device, was completely airtight. Inside were arranged three troughs side by side. From the outer ones were hung large sheets of filter paper.

The benzoates in benzene solution were applied in the normal way to a prepared strip of paper. This was hung from the centre trough at evening and the outer troughs were filled, one with methanol saturated with heptane, the other with heptane saturated with methanol. Next morning the centre trough was filled with the heptane phase through a hole (normally closed) in the chamber lid. The solvent front moves $25 \mathrm{~cm}$. in about one and a half hours.

For the dinitrobenzoates of the following alcohols, $R_{F}$ values $( \pm 0.02)$ were obtained for a run of about $25 \mathrm{~cm}$. at $20^{\circ} \mathrm{C}$. :

$\begin{array}{llll}\text { M.ethanol } & 0.24 & \text { 2-Butanol } & 0.61 \\ \text { Ethanol } & 0.39 & \text { 2-Methyl-1-propanol } & 0.55 \\ \text { 1-Propanol } & 0.46 & \text { 1-Pentanol } & 0.66 \\ \text { 2-Propanol } & 0.51 & \text { 3-Methyl-1-butanol } & 0.65 \\ \text { 1-Butanol } & 0.57 & \text { 1-Hexanol } & 0.72\end{array}$

If the values for the homologous series of derivatives of normal alcohols are used for calculating the corresponding $R_{M}$ values ${ }^{z}$ and these are plotted against the number of methylene groups in the alcohols, the points lie approximately on a straight line.

The work described in this communication was carried out as part of the programme of the Food Investigation Organization of the Department of Scientific and Industrial Research.

Ditton Laboratory

D. F. MeIGH

(Department of Scientific and Industrial Research), East Malling,

Maidstone, Kent.

Nov. 28.

${ }^{1}$ Kice, R. G., Keller, G. J., and Kirchner, J. G., Anal. Chem., 23, 194 (1951).

2 Kariyone, T., Hashimoto, V., and Kimura, M., Nature, 168, 511
(1951).

${ }^{3}$ White, J. W., and Dryden, E. C., Anal. Chem., 20, 853 (1948).

- Bate-Smith, E. C., and Westall, R. G., Biochim. et Biophys. Acta 4, $427(1950)$

\section{Feasibility of Quantitative Separation of the Threo- and Erythro-Forms of Amino- Acids by Column Chromatography}

'THн methods that are at present available for the separation of the diastereoisomeric forms of aminoacids are subject to several limitations. In the usual fractionation procedures only one of the isomers, at most, is obtained pure, the other remaining contaminated. A high degree of purity is realized only at the expense of yield. The difficulties inherent in isotopic synthesis make the above operations increasingly wasteful, thus further limiting the practicability of these methods.
We wish to report that the application of column chromatography, using an ion-exchange resin ('Dowex-50', 250,500 mesh), led to the clean-cut and quantitative separation of $d l$-threonine and $d l$ allothreonine. On the scale employed ( $3 \mathrm{mM})$, the chromatographic separation was complete, whereas chemical separation, although yielding microbiologically pure $d l$-threonine, resulted in a $d l$-allothreonine fraction containing much of the threo-form. The carbon-14 labelled products of both chemical and chromatographic separation have been tested for purity by microbiological assay with $L$. foecales.

The quantitative recovery, the completeness of the separation and the economy of effort resulting from the use of the chromatographic procedure offer great advantages. 'The investigation is being extended to other $\beta$-substituted serines and isoleucine. Details of this work will be published elsewhere.

Oliver G. Lien, JUN.

Alexander T. Shutgin

Emery M. Gat

Davio M. Greenberg

Division of Biochemistry, School of Medicine,

University of California, Berkeley, California. Nov. 30.

\section{Counter-Current Distribution of Nisins,}

IT has been suggested" that the phrase " 100 extractions" quoted from my work by $\mathrm{Hirsch}^{2}$ is liable to misinterpretation. Amplification is therefore needed.

"Extraction" was used by Bush and Densen" to describe the process occurring in each single unit (for example, separating funnel) at each stage of the counter-current distribution. With their technique, $n^{2}$ extractions are made in a chain of $n$ units, giving $n$ top layers and $n$ bottom layers. "Extraction" must therefore be distinguished from "transfer".

The technique of Bush and Densen was applied to various samples of nisin, $A$ and $L$, mentioned by Hirsch, giving the distribution shown in Fig. 1 . Batch 138 is included as an example of a preparation with two active components. "Commercial $B$ ", different from $B$ of batch 138, was distributed earlier by Craig's method ${ }^{4}$ in twenty test-tubes (actual number of extractions 210, not 200) and appeared to give two major components, both differing from $A$ and $L$; but the measured partition

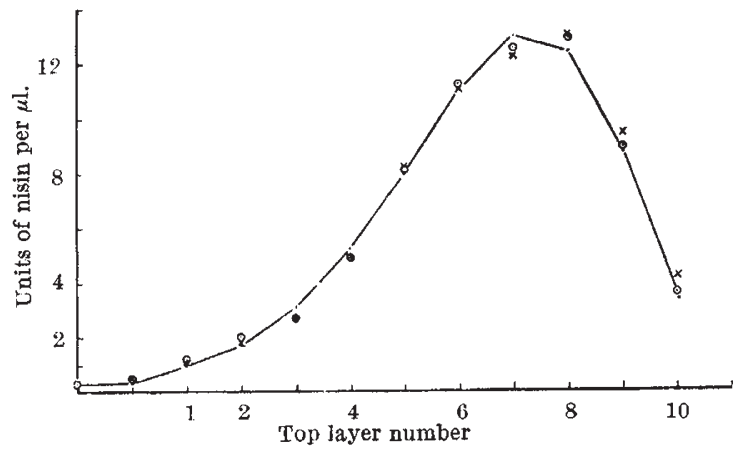

Fig. 1. Distribution of nisins $A$ and $L$

Theoretical curve for a single substance $; \odot$, experimental points for $I ; \times$, experimental points for $A$ 


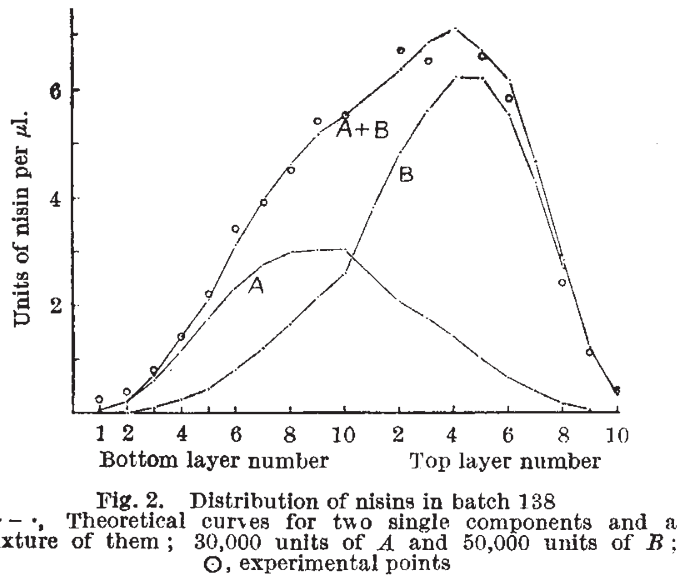

coefficients were not sufficiently constant to permit a confident analysis of the curve. The solvents were made by adding $40 \mathrm{ml}$. of glacial acetic acid and $5.44 \mathrm{gm}$. sodium chloride to $320 \mathrm{ml}$. of distilled water, adjusting the $p H$ to about 3.0 with $10 \mathrm{~N}$ sodium hydroxide, and adding to this buffer solution $40 \mathrm{ml}$. of methanol and $200 \mathrm{ml}$. $n$-butanol. The partition coefficients of the nisins depended upon the $p \mathrm{H}$ of the buffer.

N. J. BerRIDGE

National Institute for Research in Dairying,

Shinfield, Nr. Reading. Nov. 30.

${ }^{1}$ Abraham. E. P., and Newton, G. G. F. (private communication). $2 \mathrm{Hirsch}$, A., Nature, 167, 1031 (1951).

Bush, M. T., and Densen, P. M., Anal. Chem., 20, 121 (1948).

“Craig, I. C., J. Biol. Chem., 155, 519 (1944).

\section{Suggested Use of a Bismuth Mercapto- imidazole Compound as a Specific Colour Reagent for lodides}

THE reaction of bismuth salts with certain organic thio-compounds, for example, thiourea and dimercaptothiodiazole, to form coloured complexes of the metal is a well-known analytical procedure. Other workers (cf. Naiman ${ }^{1}$ ) have reported on the detection of bismuth with a mixture of 2-methylbenzothiazole and potassium iodide, which results in the formation of a coloured complex iodide. However, the use of such a reaction for the detection of iodides does not appear to have been reported, probably on the basis of specificity. In certain work on the colorimotric detection of mercaptoimidazoles, I found ${ }^{2}$ that the yellow metallic complex, formed by the interaction of bismuth sulphate (acid) and 1-methyl-2 mercaptoimidazole, reacted with iodide ions to form an intensely coloured complex iodide. Since the reaction has been found to be specific for iodides, the use of such a reagent for their detection is suggested.

The colour reagent is prepared by adding $50 \mathrm{mgm}$. of bismuth sulphate (acid) and $1 \mathrm{ml}$. of $N$ sulphuric acid to $10 \mathrm{ml}$. of a 0.1 per cent aqueous solution of 1-methyl-2-mercaptoimidazole. After mixing, the undissolved bismuth sulphate is allowed to settle, and the yellow-coloured supernatant fluid used for the test.

The addition of one or two drops of the reagent to a small crystal of an iodide results in the formation of a red coloration, which upon standing or upon agitation forms a red micro-crystalline precipitate. The sensitivity of the reaction is in the.region of $100 \mu \mathrm{gm}$. Free iodine reacts similarly; but the colour disappears on standing. Iodates and periodates do not react, nor do other metals and radicals. Metals which form insoluble sulphates give a white precipitate. The complex iodide formed in the reaction is practically insoluble in water and dilute mineral acids. As would be expected, oxidizing agents, for example, hydrogen peroxide, liberate iodine from it. When it is prepared in bulk, the red complex exhibits some degree of fluorescence, which is quenched upon shaking the reaction mixture with ethyl acetate, the resulting solution having a yellow colour.

The mercaptoimidazole ring is essential for colour formation. Other thio-compounds such as thiourea do not give the reaction. It would appear that isolation of the yellow bismuth mercaptoimidazole compound would give a much more sensitive reagent, but this has not been carried out here. Other mercaptoimidazoles were examined in this connexion. The 2-mercaptoimidazole, upon reaction with bismuth salts, did not give as sensitive a colour reagent for iodides. Likewise, the 4-aminomethyl-2-mercaptoimidazole reacted with bismuth to give a yellow compound, but this would not react with iodides.

Ronali A. MCALLISTER

Biochemical Laboratory,

Royal Samaritan Hospital, Glasgow, S.2.

Nov. 22.

' Naiman, B., J. Chem. Educ., 14, 484 (1937).

? McAllister, R. A., J. Pharm. and Pharmacol. (in the press).

\section{A New Method for the Degradation of Peptides}

THE main problem in working out the structure of peptides is to establish their amino-acid sequence. An essential part of this task consists in determining the amino-terminal and carboxyl-terminal aminoacid residues of the peptide chain (the amino-acid and the amino-acid) ${ }^{1}$. Many methods are dealing with this problem ${ }^{2}$.

We wish to report a new method, which makes it possible to determine the two terminal amino-acid residues at the amino end (the amino-acid and the neighbouring residue) in one procedure. This method is based on former investigations of one of us $\left(F . W_{.}\right)^{3}$, showing that, on alkali-treatment of $\mathrm{N}$-carbethoxydipeptides, a rearrangement takes place leading to the formation of carbonyl-bis-amino-acids.

The following scheme pictures the new degradation procedure using a tripeptide as an example.

The peptide is converted into a carbalkoxycompound (I, $R=\mathrm{CH}_{3}, \mathrm{C}_{2} \mathrm{H}_{5}$ or $\mathrm{C}_{6} \mathrm{H}_{5} \mathrm{CH}_{2}$ ), which on heating with two moles of dilute alkali gives rise to the urea derivative (III), the hydantoin (II) being probably formed as an intermediate. After treatment with hydrochloric acid, the amino-acids (1) and (2) are easily split off in the form of the hydantoin (IV, V), which may be separated from amino-acid (3)-and the following amino-acids in the case of higher peptides-by means of extraction with ether.

After hydrolysis of the hydantoin (IV, V) the constituent amino-acids (1) and (2) can be identified by paper chromatography. Conversion of the hydantoin (IV) to the acid chloride, reduction of the 